\title{
entornos virtuales 2D y \\ 3D. Un análisis con \\ estudiantes del grado \\ de ingeniería
}

2015 num. 1. Pag. 90-93

ISSN 1135-1438. EISSN 2385-4731

http://revistes.publicacionsurv.cat/index.php/ute

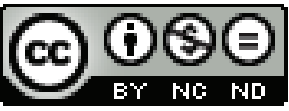

\section{Byron Ernesto Vaca Barahona ${ }^{a}$}

Data de defensa: 20/05/2015

Palabras clave: comunicación, interacción, interactividad.

\section{Justificación}

Las tecnologías de información y comunicación se han integrado de manera exitosa en las diferentes actividades querealizamos a diario. La educación virtual es un área muy importante que ha encontrado en las tecnologías de la información ycomunicación una alternativa válida para su desarrollo. Sin embargo en el contexto formativo, el desarrollo y cambio tecnológico acelerado impulsa recurrentemente el desarrollo deinvestigaciones que validen, adapten y evalúen teorías, metodologías, técnicas y herramientas para garantizar su integración demanera adecuada en busca de alcanzar la calidad académica deseada. Con el propósito de afianzar la calidad de la educación virtual, se debe profundizar en la investigación de elementos de carácter multidisciplinario relacionados con la psicología, la sociología, la didáctica y la comunicación, aspectos relacionados con eldesarrollo tecnológico y con el uso de una metodología didáctica propia. Desde nuestra perspectiva sentimos que es necesario profundizar en la investigación de los aspectos comunicacionales en losprocesos formativos, puesto que el aprender depende en gran medida de su efectividad y eficiencia. Con este propósito, se estudia la Interacción y la Interactividad mediada pedagógicamente en entornos virtuales de aprendizaje (EVA) 2D y 3D, buscando determinar qué plataforma auspicia de mejor manera el desarrollo de los procesos comunicacionales. El objetivo general de este trabajo de investigación se concreta en: Analizar los procesos comunicativos desde la perspectiva dela interacción y la interactividad en entornos virtuales de aprendizaje 2D y 3D con estudiantes

\footnotetext{
${ }^{a}$ Escuela Superior Politécnica de Chimborazo
} 
universitarios. Este objetivo general de análisis se concreta en los siguientes objetivos específicos:

Analizar la interacción intrapersonal, interpersonal e intragrupal generada por los estudiantes en el contexto de una actividadacadémica en los entornos 2D y 3D.

Describir la percepción de la experiencia comunicativa de los estudiantes en el contexto de la actividad académica, considerando la interacción y la interactividad en los entornos $2 \mathrm{D}$ y $3 \mathrm{D}$.

\section{Metodología}

El presente trabajo se desarrollará bajo una aproximación metodológica cualitativa dado que analiza de manera exhaustivainformación referida a casos concretos, lo que nos permite hacer una descripción de la realidad observada a profundidad. No obstante, combina estrategias de recogida y análisis de datos tanto cualitativos como cuantitativos. En la investigación se observa la actividad de 32 estudiantes universitarios, 16 de ellos trabajaron en un entorno virtual deaprendizaje 2D, concretamente Moodle y otros 16 lo hicieron en un entorno virtual de aprendizaje 3D, OpenSim. Se estudia el contenido del discurso de las interacciones de los estudiantes, aplicando un proceso de análisis e interpretaciónque permita clasificarlas en las cataegorías de intrapersonal, interpersonal e intragrupal. A continuación se estudian los discursos de las interacciones de los estudiantes, tomando como referencia las categorías ysubcategorías planteadas en la técnica de análisis semiótico conocida como Apreciación Teórica. Se analizan, interpretan y seclasifican estás interacciones en Actitudes y Aptitudes evidenciadas en la comunicación generada al desarrollar la actividadacadémica. Se cuantifican las interacciones clasificadas en las diferentes categorías y se describen los resultados observados con elpropósito de determinar qué entorno favorece mejor la comunicación mediada pedagógicamente. Adicionalmente, se diseñó un instrumento para que los estudiantes evaluaran su "experiencia comunicacional mediadapedagógicamente en el entorno 2D de Moodle y en el entorno 3D de OpenSim", resultados que son agrupados, analizados ydescritos según lo observado. Con estos resultados cuantitativos, se aplica la prueba paramétrica de CHI CUADRADO paradeterminar la existencia de correlación entre la autopercepción de la experiencia comunicacional de los estudiantes y el entornovirtual en el que trabajaron.

\section{Conclusiones}

En relación al estudio de los discursos para clasificar el tipo de interacción:

- El docente debe dimensionar adecuadamente la carga de trabajo que conlleva desarrollar la actividad académica, esconveniente dividir el desarrollo de la actividad académica en sub - actividades más pequeñas con el propósito de partir la cargade trabajo en el tiempo planificado para su desarrollo. 
- Al diseñar la actividad académica se debe dimensionar el tamaño y la complejidad del trabajo, es recomendable dividir eltrabajo en actividades cortas y alcanzables en ciclos de interacción cortos, la sensación repetida de éxito en los estudiantes alresolver actividades más pequeñas, permite mantener niveles importantes de automotivación.

- En cuanto a la conformación de grupos de trabajo, es importante integrar a estudiantes con diferentes perfiles de aprendizaje. Esta integración favorece la diversidad en las preferencias o formas de aprender, lo que enriquece el debate de los miembrosdel grupo de trabajo generando procesos comunicacionales basados en interacción con el propósito de colaborar.

- Se observa que el entorno virtual de aprendizaje 3D de OpenSim favorece de mejor manera los procesos comunicacionalesbasados en interacción.

En relación al estudio de los discursos para el sistema de Actitudes y Aptitudes:

- $\quad$ En este proceso se observa el perfeccionamiento del SER reflejado en las actitudes de felicidad, seguridad, satisfacción, reconocimiento, propiedad, ofrecer. Pero además el perfeccionamiento de los SABERES reflejados en las aptitudes de preguntar, responder, organizar, evaluar y mandar. Tanto el SER como el SABER son objetivos deseados en los procesos de formación de los ciudadanos.

- Los docentes debemos estar conscientes en la importancia de construir ambientes formativos donde el desarrollo decompetencias haga a los estudiantes aptos para resolver problemas reales. Pero además es muy importante que en losprocesos formativos reforcemos la formación del ser humano con con actitudes positivas al momento de colaborar en el desarrollo de actividades grupales.

- El diseño de la actividad académica debe considerar la generación de espacios de reflexión interna en cada estudiante, pero además debe fomentar la participación grupal. Tanto la reflexión individual y grupal consolidan los saberes a través de procesos de retroalimentación y debate al mismo tiempo que se fortalece el ser al compartir espacios de trabajo con los miembros del grupo.

En relación con la encuesta "Análisis subjetivo de percepciones de la experiencia comunicativa en el entorno virtual deaprendizaje":

- - Los docentes deben asumir el reto de innovar, integrando tecnologías de la información y comunicación renovadas que sean coherentes a las nuevas realidades sociales de los estudiantes, promoviendo la interacción natural a través de espacios virtuales de aprendizaje que promuevan su criticidad constructiva y participativa.

- $\quad$ - Los entornos de simulación 3D y la presencialidad virtual de los actores del proceso de enseñanza - aprendizaje a través de sus avateres, eliminan las barreras 
del formalismo existente entre docente y estudiante, lo que permite el desarrollo natural de la comunicación entre pares, facilitando el debate y la colaboración en los procesos de aprender. 